\title{
Geometric phases causing lifetime modifications of metastable states of hydrogen
}

\author{
M.-I. Trappe, ${ }^{1,2, *}$ P. Augenstein, ${ }^{3, \dagger}$ M. DeKieviet, ${ }^{3, \dagger}$ T. Gasenzer, ${ }^{2,4,5, \S}$ O. Nachtmann ${ }^{2, \mathbb{I}}$ \\ ${ }^{1}$ Centre for Quantum Technologies, National University of Singapore, 3 Science Drive 2, Singapore 117543, Singapore \\ ${ }^{2}$ Institut für Theoretische Physik, Universität Heidelberg, Philosophenweg 16, 69120 Heidelberg, Germany \\ ${ }^{3}$ Physikalisches Institut, Universität Heidelberg, Im Neuenheimer Feld 226, 69120 Heidelberg, Germany \\ ${ }^{4}$ Kirchhoff-Institut für Physik, Universität Heidelberg, Im Neuenheimer Feld 227, 69120 Heidelberg, Germany \\ ${ }^{5}$ ExtreMe Matter Institute EMMI, GSI Helmholtzzentrum für Schwerionenforschung, Planckstraße 1, 64291 Darmstadt, Germany
}

(13th September 2018)

\begin{abstract}
Externally applied electromagnetic fields in general have an influence on the width of atomic spectral lines. The decay rates of atomic states can also be affected by the geometry of an applied field configuration giving rise to an imaginary geometric phase. A specific chiral electromagnetic field configuration is presented which geometrically modifies the lifetimes of metastable states of hydrogen. We propose to extract the relevant observables in a realistic longitudinal atomic beam spin-echo apparatus which allows the initial and final fluxes of the metastable atoms to be compared with each other interferometrically. A geometry-induced change in lifetimes at the $5 \%$-level is found, an effect large enough to be observed in an available experiment.
\end{abstract}

PACS numbers: 03.65.Vf, 03.75.Dg, 32.70.Cs, 37.25.+k

\section{Introduction}

[Atoms being exposed to an adiabatically varying external field can acquire geometric phases [1,2]. For metastable states, such geometric phases are in general complex. The imaginary part of such a phase influences the lifetime, see e.g. [3-6].

In Refs. [7-11], we have presented studies of geometric phases for metastable states of hydrogen. Both, parity-conserving (PC) and parity-violating (PV) geometric phases were identified. It was, in particular, shown in [11] that the lifetimes of metastable $2 \mathrm{~S}$ hydrogen states can be influenced by geometric phases acquired by the atom in suitable external electric and magnetic fields. A concrete example of the influence of a complex geometric phase on the lifetime of atomic states was discussed in [11]. With the field configurations investigated there geometric effects on the lifetimes at the per mille level were found.

\footnotetext{
*martin.trappe@quantumlah.org

† augenstein@physi.uni-heidelberg.de

${ }^{\ddagger}$ maarten.dekieviet@physik.uni-heidelberg.de

$\S_{\text {t.gasenzer@uni-heidelberg.de }}$

๑O.Nachtmann@thphys.uni-heidelberg.de
}

In the present paper we shall explore suitable field configurations which lead, in theory, to geometric effects on the lifetimes of metastable hydrogen states up to the level of several per cent. We propose to measure the lifetime shifts by means of an existing longitudinal atomic beam spin-echo interferometer that allows the initial and final fluxes of metastable atoms to be compared with each other. The results presented here were obtained by means of the theoretical formalism introduced in detail in Refs. [9. 11]. We refer to these papers for the discussion of the general context of our investigations and of the proposed experimental scheme, as well as for many further references. We will, in particular, make use of specific expressions and formulae from these papers, referring to them without repeating their derivations.

\section{Metastable hydrogen in the longitudinal atomic beam spin-echo apparatus}

\subsection{Atomic-beam spin-echo interferometer}

As in [9] we consider metastable $2 \mathrm{~S}$ hydrogen states in the spin-echo interferometer described in [12]. Figure 1 shows a schematic view of the atomic-beam spin- 


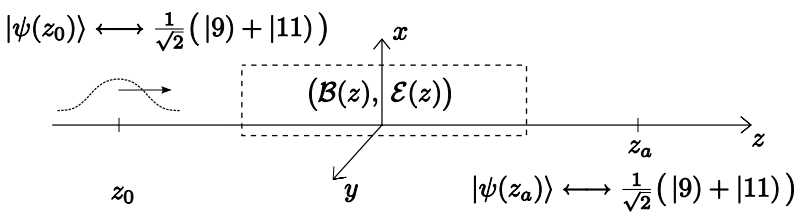

Figure 1: Scheme of the atom interferometry experiment. The atom is prepared around $z_{0}$ and analysed around $z_{a}$. We start with a superposition $\left|\psi\left(z_{0}\right)\right\rangle$ of the two states $\left.\mid 9\right)$ and $\left.\mid 11\right)$. After passing the electric and magnetic fields the wave function is projected onto an analysing state $\left|\psi\left(z_{a}\right)\right\rangle$, for example, again onto a superposition of the states $\left.\mid 9\right)$ and $\mid 11)$. The coordinate axes used, $x, y, z$, indexed in the formulae as 1,2 , and 3 , respectively, are also indicated.

echo interferometer. An atomic state, in general a superposition of local energy eigenstates, enters the interferometer at $z_{0}$. The state is then subjected to electric and magnetic fields $\mathcal{E}(z)$ and $\mathcal{B}(z)$. Finally, it is analysed at $z_{a}$ by projection on a chosen final state. In reference to the experiment, we set in the following

$$
\begin{aligned}
& z_{0}=0 \mathrm{~m}, \\
& z_{a}=0.66 \mathrm{~m} .
\end{aligned}
$$

First we consider field configurations of a general type, consisting of two regions I and II in space and/or time of the spin-echo setup [12], in which the spins precess forward and backwards, respectively (thus separated by an effective $\pi$-pulse). These regions have an electric field

$$
\begin{aligned}
\mathcal{E}(z)= & \mathcal{E}_{\mathrm{I}}(z) \Theta\left(\frac{1}{2} z_{a}-z\right) \Theta(z) \\
& +\mathcal{E}_{\mathrm{II}}(z) \Theta\left(z_{a}-z\right) \Theta\left(z-\frac{1}{2} z_{a}\right),
\end{aligned}
$$

and a magnetic field with the components

$$
\mathcal{B}(s ; z)=\mathbf{e}_{1} \mathcal{B}_{1}(z)+\mathbf{e}_{2} \mathcal{B}_{2}(z)+\mathbf{e}_{3} \mathcal{B}_{3}(s ; z),
$$

where

$$
\begin{aligned}
\mathcal{B}_{i}(z)= & \mathcal{B}_{i \mathrm{I}}(z) \Theta\left(\frac{1}{2} z_{a}-z\right) \Theta(z) \\
& +\mathcal{B}_{i \mathrm{II}}(z) \Theta\left(z_{a}-z\right) \Theta\left(z-\frac{1}{2} z_{a}\right)
\end{aligned}
$$

for $i=1,2$, and

$$
\begin{aligned}
\mathcal{B}_{3}(s ; z)= & \mathcal{B}_{3 \mathrm{I}}(z) \Theta\left(\frac{1}{2} z_{a}-z\right) \Theta(z) \\
& +s \mathcal{B}_{3 \mathrm{II}}(z) \Theta\left(z_{a}-z\right) \Theta\left(z-\frac{1}{2} z_{a}\right) .
\end{aligned}
$$

We also require

$$
\begin{aligned}
\mathcal{E}(0) & =\mathcal{E}\left(\frac{1}{2} z_{a}\right)=\mathcal{E}\left(z_{a}\right)=0, \\
\mathcal{B}(s ; 0) & =\mathcal{B}\left(s ; \frac{1}{2} z_{a}\right)=\mathcal{B}\left(s ; z_{a}\right)=0 .
\end{aligned}
$$

In (2)- (5) $\Theta(\cdot)$ is the usual step function and $s$ is a parameter, which acts as a detuning between the spin precession regions I and II, and is varied around the spin echo point, $s=1$, by typically

$$
0.4 \leq s \leq 1.6
$$

The variation of $s$, that is, the variation of the magnetic field $\mathcal{B}_{3}$ in the second half of the interferometer produces the oscillations in the spin-echo signal; see [9]. Explicit examples of external fields within this general form are given in Section 3 below (see Figures 24).

An atom travelling through the interferometer with field configuration (2)-6) traces out, in parameter space, a closed path $C_{s}$, where $s$ is kept fixed. In fact, $C_{s}$ is composed of two successive paths in regions I and II,

$$
C_{s}=C_{\mathrm{I}}+C_{\mathrm{II}, s} .
$$

We shall now consider field configurations that, in parameter space, correspond to oppositely oriented paths, either along the reverse of the complete path $C$, or along the reverse of the paths $C_{\mathrm{I}}$ and $C_{\mathrm{II}}$ separately.

For reversing the complete path $C$ we consider the fields

$$
\begin{aligned}
\mathcal{E}^{\text {rev }}(z)= & \mathcal{E}\left(z_{a}-z\right), \\
\mathcal{B}_{i}^{\text {rev }}(z)= & \mathcal{B}_{i}\left(z_{a}-z\right), \quad \text { for } i=1,2 \\
\mathcal{B}_{3}^{\text {rev }}(s ; z)= & s \mathcal{B}_{3 \mathrm{II}}\left(z_{a}-z\right) \Theta\left(\frac{1}{2} z_{a}-z\right) \Theta(z) \\
& +\mathcal{B}_{3 \mathrm{I}}\left(z_{a}-z\right) \Theta\left(z_{a}-z\right) \Theta\left(z-\frac{1}{2} z_{a}\right) .
\end{aligned}
$$

From (2)- (6) and (9) we see that, in the reverse field configuration, the atomic system traces out the path which is the reversed one of (8),

$$
\bar{C}_{s}=\bar{C}_{\mathrm{II}, s}+\bar{C}_{\mathrm{I}}
$$

Note that for the reverse field configuration the magnetic field component $\mathcal{B}_{3}$ is varied with $s$ in the first half of the interferometer.

For the second case of reversing the paths in regions I and II of the interferometer separately, we consider the 
following fields:

$$
\begin{aligned}
\tilde{\mathcal{E}}^{\text {rev }}(z)= & \mathcal{E}_{\mathrm{I}}\left(\frac{1}{2} z_{a}-z\right) \Theta\left(\frac{1}{2} z_{a}-z\right) \Theta(z) \\
& +\mathcal{E}_{\mathrm{II}}\left(\frac{3}{2} z_{a}-z\right) \Theta\left(z_{a}-z\right) \Theta\left(z-\frac{1}{2} z_{a}\right) \\
\tilde{\mathcal{B}}_{i}^{\text {rev }}(z)= & \mathcal{B}_{i \mathrm{I}}\left(\frac{1}{2} z_{a}-z\right) \Theta\left(\frac{1}{2} z_{a}-z\right) \Theta(z) \\
& +\mathcal{B}_{i \mathrm{II}}\left(\frac{3}{2} z_{a}-z\right) \Theta\left(z_{a}-z\right) \Theta\left(z-\frac{1}{2} z_{a}\right), \\
\text { for } i= & 1,2 \\
\tilde{\mathcal{B}}_{3}^{\text {rev }}(s ; z)= & \mathcal{B}_{3 \mathrm{I}}\left(\frac{1}{2} z_{a}-z\right) \Theta\left(\frac{1}{2} z_{a}-z\right) \Theta(z) \\
& +s \mathcal{B}_{3 \mathrm{II}}\left(\frac{3}{2} z_{a}-z\right) \Theta\left(z_{a}-z\right) \Theta\left(z-\frac{1}{2} z_{a}\right) .
\end{aligned}
$$

Here the path of the atom in parameter space in relation to $(8)$ is

$$
\bar{C}_{s}^{\prime}=\bar{C}_{\mathrm{I}}+\bar{C}_{\mathrm{II}, s} .
$$

\subsection{Hydrogen spin-echo observables}

The hydrogen states under investigation are $2 \mathrm{~S}$ states that are admixed with $2 \mathrm{P}$ states in external electric fields. Our numbering of the $16(n=2)$-states of hydrogen is explained in detail in Appendix A, Table A.2, of [11]. The index set of metastable states is

$$
I=\{9,10,11,12\}
$$

The initial state at $z=z_{0}$ is a superposition of metastable states

$$
\begin{aligned}
\left.|\psi(0)\rangle\right|_{\text {internal }} & \left.=\sum_{\alpha \in I} c_{\alpha} \mid \alpha\left(z_{0}\right)\right), \\
\sum_{\alpha \in I}\left|c_{\alpha}\right|^{2} & =1 .
\end{aligned}
$$

See (72) in [9] for the complete state vector. Here and in the following we write out only the internal part of it. In (14) and in the following $\mid \alpha(z))(\alpha=1, \ldots, 16)$ are the local energy right eigenstates corresponding to the fields $\mathcal{E}(z), \mathcal{B}(z)$; see (13) of [9].

As discussed in [9], the effective potentials $\mathcal{V}_{\alpha}(z)$ entering the Schrödinger equation for the atomic states in the external fields are not equal to the local complex energy eigenvalues $E_{\alpha}(z)$, see (31)-(33) of [9], as they include additional geometric-phase effects. But, as we shall show below, in our case this difference is negligible. Nonetheless, we work in the following with the effective potentials as this is the correct procedure. The value of the effective potential for the state $\alpha$ at point $z$ is in general complex

$$
\mathcal{V}_{\alpha}(z)=\operatorname{Re} \mathcal{V}_{\alpha}(z)-\frac{\mathrm{i}}{2} \Gamma_{\alpha}(z) .
$$

Here

$$
\Gamma_{\alpha}(z)=-2 \operatorname{Im} \mathcal{V}_{\alpha}(z)
$$

is the local decay rate of the state $\alpha$; see (32), (33) of [9]. For the field configurations considered in the present work, we find for $\alpha=9,11$

$$
\left|\operatorname{Re}\left(\mathcal{V}_{\alpha}(z)-E_{\alpha}(z)\right)\right| \lesssim 10^{-16} \mathrm{eV},
$$

and

$$
\frac{\left|\operatorname{Im}\left(\mathcal{V}_{\alpha}(z)-E_{\alpha}(z)\right)\right|}{\max _{0 \leq z \leq z_{\alpha}}\left|\operatorname{Im} E_{\alpha}(z)\right|} \lesssim 10^{-10},
$$

that is, the numerical differences between $\mathcal{V}_{\alpha}(z)$ und $E_{\alpha}(z)$ are negligible since we shall deal with energies at the $\mu \mathrm{eV}$ scale; cf. Figure 6 below.

The atoms in the beam have typical longitudinal velocity $v_{z}$, wave number $k_{z}$ and de Broglie wavelength $\lambda$ (see (20) of [9])

$$
\begin{aligned}
v_{z} & =\frac{k_{z}}{m} \approx 3500 \mathrm{~m} / \mathrm{s}, \\
k_{z} & \approx 5.6 \times 10^{10} \mathrm{~m}^{-1}, \\
\lambda & =\frac{2 \pi}{k_{z}} \approx 1.1 \times 10^{-10} \mathrm{~m} .
\end{aligned}
$$

At the end of the interferometer, at $z=z_{a}$, the atomic state is projected onto a chosen state (see (90) of [9])

$$
\begin{aligned}
\mid p) & \left.=\sum_{\alpha \in I} p_{\alpha} \mid \alpha(0)\right), \\
\sum_{\alpha \in I}\left|p_{\alpha}\right|^{2} & =1 .
\end{aligned}
$$

The integrated flux $\mathcal{F}_{p}$ for this state is the experimental observable

$$
\begin{gathered}
\mathcal{F}_{p}=\sum_{\alpha, \beta \in I} p_{\beta} p_{\alpha}^{*} c_{\beta}^{*} c_{\alpha} \exp \left[-\left(\Delta \tau_{\beta}-\Delta \tau_{\alpha}\right)^{2} /\left(8 \sigma_{k}^{\prime 2}\right)\right] \\
\times U_{\beta}^{*}\left(z_{a}, z_{0} ; \bar{k}_{m}\right) U_{\alpha}\left(z_{a}, z_{0} ; \bar{k}_{m}\right) .
\end{gathered}
$$

All quantities occuring in (21) are defined and explained in the context of Eq. (105) in [9]. We briefly recall them in the following. 
The $U_{\alpha}$ contain the dynamic and geometric phases, see (101) of [9],

$$
U_{\alpha}\left(z_{a}, z_{0} ; \bar{k}_{m}\right)=\exp \left[-\mathrm{i} \varphi_{\alpha}\left(z_{a}\right)+\mathrm{i} \gamma_{\alpha}\left(z_{a}\right)\right] .
$$

Here $\bar{k}_{m}$ is the peak value of the wave-number distribution in the wave packet; see (78), (79) of [9]. The $\Delta \tau_{\alpha, \beta}$ are the shifts of the reduced arrival times as defined in (99) of [9]. The dynamic and geometric phases acquired by the state with label $\alpha$ from $z=0$ to $z$ are denoted by $\varphi_{\alpha}(z)$ and $\gamma_{\alpha}(z)$, respectively. We have

$$
\begin{aligned}
& \varphi_{\alpha}(z)=\frac{1}{v_{z}} \int_{0}^{z} \mathrm{~d} z^{\prime} \mathcal{V}_{\alpha}\left(z^{\prime}\right), \\
& \gamma_{\alpha}(z)=\mathrm{i} \int_{0}^{z} \mathrm{~d} z^{\prime}\left(\widetilde{\alpha\left(z^{\prime}\right) \mid} \frac{\partial}{\partial z^{\prime}} \mid \alpha\left(z^{\prime}\right)\right),
\end{aligned}
$$

where $(\widetilde{\alpha(z)} \mid$ are the local energy left eigenstates. Note that we use a slightly different notation here, as compared to [9]. To obtain [22] from (101)-(103) of [9] the following replacements have to be made

$$
\begin{aligned}
\phi_{\mathrm{dyn}, \alpha} & \rightarrow \varphi_{\alpha}\left(z_{a}\right), \\
\phi_{\text {geom }, \alpha} & \rightarrow \gamma_{\alpha}\left(z_{a}\right) .
\end{aligned}
$$

The main quantities of interest to us here are the effective decay rates of the metastable states, see (127) of [11], which depend on the path $C$ in parameter space. For a state $\alpha \in I$, these decay rates, multiplied by the flight time $T$ from $z_{0}$ to $z_{a}$, are given by

$$
T \Gamma_{\alpha, \mathrm{eff}}(C)=-2 \operatorname{Im} \varphi_{\alpha}\left(z_{a}\right)+2 \operatorname{Im} \gamma_{\alpha}\left(z_{a}\right) .
$$

The dynamic contribution to $T \Gamma_{\alpha, \text { eff }}$ can be written as

$$
\begin{aligned}
-2 \operatorname{Im} \varphi_{\alpha}\left(z_{a}\right) & =-\frac{2}{v_{z}} \int_{0}^{z_{a}} \mathrm{~d} z \operatorname{Im} \mathcal{V}_{\alpha}(z) \\
& =\frac{m}{\bar{k}_{m}} \int_{0}^{z_{a}} \mathrm{~d} z \Gamma_{\alpha}(z)
\end{aligned}
$$

and thus depends inversely on $v_{z}$ and $\bar{k}_{m}$, respectively. In (27) $m$ denotes the hydrogen mass. In contrast, the geometric contribution in 26 ,

$$
2 \operatorname{Im} \gamma_{\alpha}\left(z_{a}\right),
$$

is independent of $v_{z}$. This different dependence on $v_{z}$ allows us to experimentally distinguish between the dynamic and geometric contributions to $T \Gamma_{\alpha, \text { eff }}$. For our setup the flight time is

$$
T=\frac{z_{a}}{v_{z}} \approx \frac{0.66}{3500} \mathrm{~s} \approx 0.2 \mathrm{~ms} .
$$

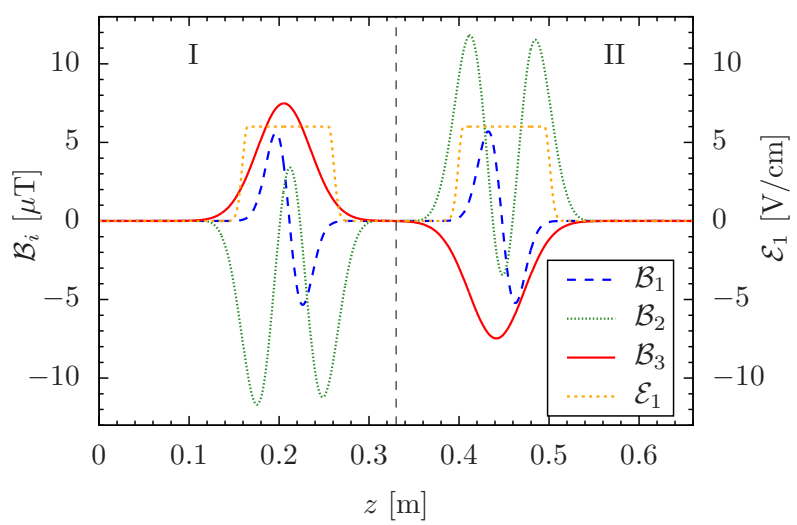

Figure 2: The functions $z \mapsto \mathcal{E}_{1}(z)$ and $z \mapsto \mathcal{B}(s ; z)$ with $s=1$, see Appendix $\mathrm{B}$ for details. Ideally, $\mathcal{B}^{\text {rev }}(1, z)=-\mathcal{B}(1 ; z)$. Regions I and II are separated by $z=z_{a} / 2$.

\section{Geometric-phase induced lifetime modification}

\subsection{Exemplary field configuration}

In the following we shall discuss a concrete example of field configurations (2)-(6) and their reverse ones, (9), and calculate the corresponding effective decay rates of metastable $\mathrm{H}$ states. We consider the fields shown in Figure 2 (for $s=1$ ) leading to the path $C$ in parameter space. The magnetic part of $C$ is illustrated in Figure 3 . We are looking here for a lifetime shift, that is, a parity conserving (PC), or even effect. We will, therefore, in the following and other than in our previous work [7-111], neglect the very small parity violating $(\mathrm{PV})$ interaction for the hydrogen atom. Hence, in all formulae taken from [9] and [11], we leave out the PV contributions.

As initial and as analysing state we choose the same superposition of the states 9 and 11:

$$
\begin{aligned}
& c_{9}=c_{11}=\frac{1}{\sqrt{2}}, c_{10}=c_{12}=0 ; \\
& p_{9}=p_{11}=\frac{1}{\sqrt{2}}, p_{10}=p_{12}=0 .
\end{aligned}
$$

The results shown in the following have been obtained with the help of the numerical software QABSE [13, 14]. The exemplary path $C$ which we choose in agreement with Eqs. (2)-(6), represents an external field configuration with electric field components $\mathcal{E}_{1} \neq 0, \mathcal{E}_{2}=\mathcal{E}_{3}=0$ and magnetic components $\mathcal{B}_{i} \neq 0(i=1,2,3)$. We con- 


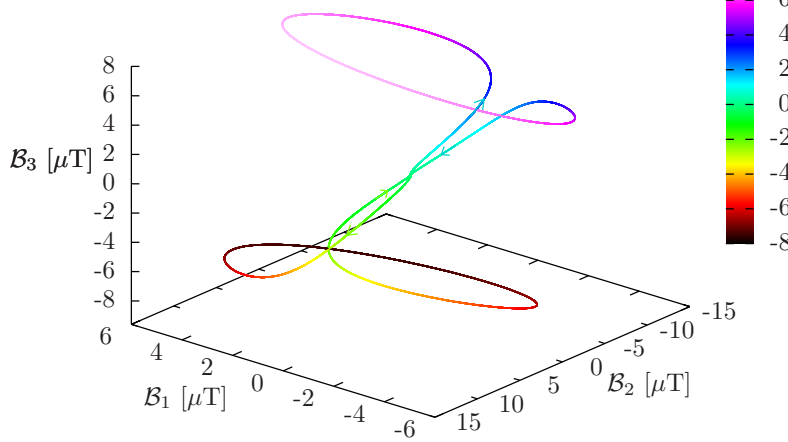

Figure 3: The path $z \mapsto \mathcal{B}(1 ; z)$ in magnetic field space, starting and ending at $\mathcal{B}=\mathbf{0}$ for $z=0$ and $z_{a}$, respectively. The values of $\mathcal{B}_{3}(1 ; z)$ are color-encoded. Also $\mathcal{E}_{1}(z)$ varies with $z$ as shown in Figure 2 and discussed in the text. The orientation of the path is chosen such that the imaginary parts of the geometric phases are maximised, given the experimental constraints to the magnetic field coils currently available.

sider the case where for $s=1$ we have

$$
\begin{aligned}
\mathcal{E}_{1}(z) & =\mathcal{E}_{1}\left(z_{a}-z\right) \\
\mathcal{B}(1 ; z) & =-\mathcal{B}\left(1 ; z_{a}-z\right) .
\end{aligned}
$$

That is, we choose $\mathcal{E}_{1}(z)$ to be a symmetric function and $\mathcal{B}(1, z)$ to be an antisymmetric function under a reflection at the point $z=z_{a} / 2$.

In Figures 2 and 3 we plot the components of these fields as functions of $z$. These fields are inspired by the realistic design of an actual experimental device, using a fit to calculated and measured field values. The electric field is given in units of $\mathrm{V} / \mathrm{cm}$ while the magnetic field components are specified in units of $\mu$ Tesla. The specific fit functions are listed in Appendix B. We emphasise that these realistic fields satisfy the symmetry conditions (31) only to a certain accuracy. We choose the electric field such that $\mathcal{E}_{1}(z)=\mathcal{E}_{1}\left(z_{a}-z\right)$. The magnetic field is produced by fixed coils, in the regions I and II of the apparatus, one for $\mathcal{B}_{3}$ and one for $\mathcal{B}_{1}$ and $\mathcal{B}_{2}$. The magnetic fields can be varied by changing the currents through these coils. We illustrate the deviations of our field configuration from the ideal symmetric setup (31) in Figure 4. In addition to the small violations of (31) by the fit functions of Appendix B we have introduced, by hand, a violation of (31) by shifting the $z$-component of the magnetic field along the beam axis (dashed line). As a measure of deviation we use

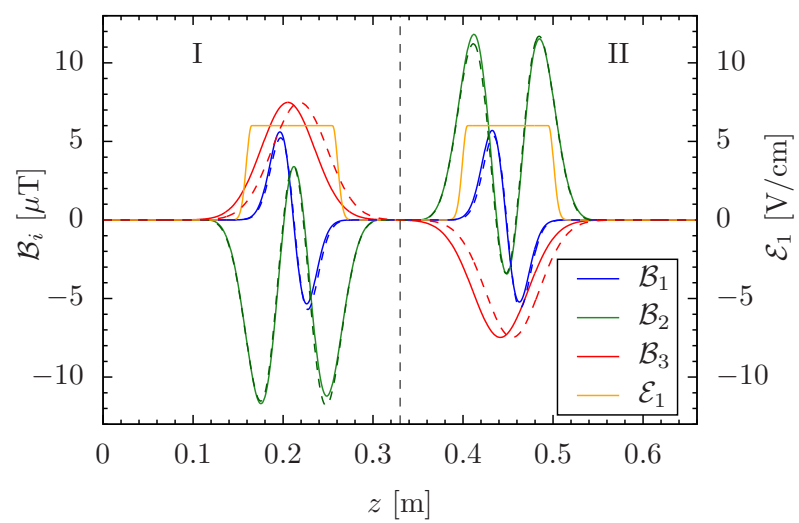

Figure 4: Illustration of the deviations of our experimentally motivated field configuration from an ideal configuration satisfying the symmetry conditions 31. The solid lines correspond to the configuration from Figure 2 while the dashed lines indicate the reversed fields, with the sign of the magnetic field switched for presentational purposes, i. e., $-\mathcal{B}^{\text {rev }}$.

$$
\Delta=\frac{1}{z_{a}} \int_{0}^{z_{a}} \mathrm{~d} z\left\{\sum_{i=1}^{3}\left[b_{i}(z)\right]^{2}\right\}^{1 / 2}
$$

where

$$
b_{i}(z)=\frac{\mathcal{B}_{i}(1 ; z)+\mathcal{B}_{i}\left(1 ; z_{a}-z\right)}{\max _{0 \leq z \leq z_{a}} \mathcal{B}_{i}(1 ; z)} .
$$

$\Delta$ vanishes if (31) holds. For the field configuration in Figure 2 the deviation 32 turns out to be $\Delta \approx 8.4 \%$ and is mainly due to the asymmetry of $\mathcal{B}_{3}$. Note that we deliberately choose the deviations 32 , here almost an order of magnitude larger than in the actual experiment, in order to demonstrate in the following the robustness of our method to this kind of experimental imperfection.

The reverse (9) of the ideal field configuration (31), for $s=1$, is obtained by leaving the electric field unchanged and reversing the current through the coils generating the magnetic field,

$$
\begin{aligned}
\mathcal{E}_{1}^{\text {rev }}(z) & =\mathcal{E}_{1}(z), \\
\mathcal{B}^{\text {rev }}(1 ; z) & =-\mathcal{B}(1 ; z) .
\end{aligned}
$$

While the parameter space in our example is fourdimensional, spanned by $\mathcal{E}_{1}, \mathcal{B}_{1}, \mathcal{B}_{2}, \mathcal{B}_{3}$, we can illustrate the projection of the path into the three-dimensional space of the magnetic fields. Figure 3 shows this projection of the path $C_{s}(8)$ for $s=1$. The corresponding $z$-dependence of $\mathcal{E}_{1}(z)$ is as shown in Figure 2. That is, $\mathcal{E}_{1}(z)$ starts at zero and is positive when $\mathcal{B}(z)$ traces out the upper loop in Figure 3 . After this, $\mathcal{E}_{1}(z)$ goes to zero 

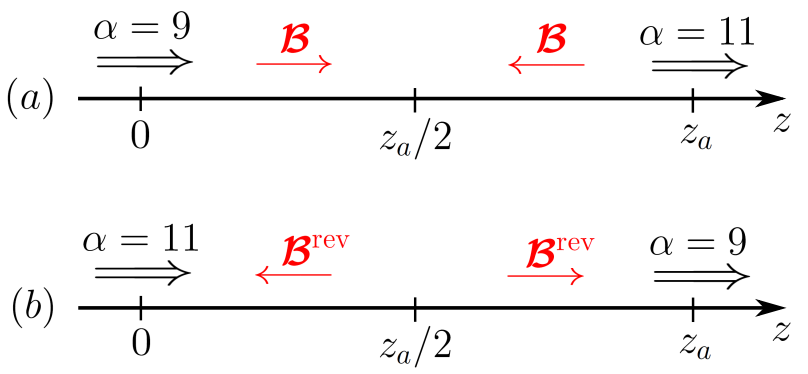

Figure 5: Illustration of the renumbering of states in the case that only $\mathcal{B}_{3}(s ; z) \neq 0$. The double line arrows indicate the spin directions. In (a) the state starting at $z=0$ with label $\alpha=9$ is subject to the path $C_{s}$ in parameter space and arrives with label $\alpha=11$. In the reverse field configuration (b) the corresponding state to start with has label $\alpha=11$ and is relabeled as $\alpha=9$ for $z>z_{a} / 2$.

at $z=z_{a} / 2$ before becoming positive again while $\mathcal{B}(z)$ traces out the lower loop in Figure 3 . Finally, both $\mathcal{E}_{1}(z)$ and $\mathcal{B}(z)$ go back to zero before ending at $z=z_{a}$.

The evolution of the states in the interferometer should be adiabatic wherever geometric phases are picked up for $0<z<z_{a} / 2$ and $z_{a} / 2<z<z_{a}$. We have made sure that this is true for all cases considered; see Appendix A. The point $z=z_{a} / 2$ is special since there we have $\mathcal{E}=0$ and $\mathcal{B}=0$ as required in 6 , implying a degeneracy to appear at this point. Making use of the numbering scheme as explained in Appendix A of [11] we find that a state with label $\alpha=9(\alpha=11)$ entering from $z<z_{a} / 2$ will have the label $\alpha=11(\alpha=9)$ for $z>z_{a} / 2$. Hereby, we make sure that the phases of the states are continuous for $z=z_{a} / 2$ despite their renumbering. In the following we shall, therefore, label the states, energies, etc., with $9 ; 11$ and $11 ; 9$ where the first/second number corresponds to the label $\alpha$ in the first/second half of the interferometer. Note that for the states $\alpha=10$ and 12 there is no relabelling at $z=z_{a} / 2$. Note furthermore that, when switching from the path defined by the fields (2), (3) to the reverse path (9), we have to compare the states $9 ; 11$ with $11 ; 9$ and, correspondingly, 11;9 with $9 ; 11$. This becomes particularly clear if in (2), (3) we consider a path with only $\mathcal{B}_{3}(s ; z) \neq 0$, of the form shown in Figure 2 and with $\mathcal{B}_{3}^{\text {rev }}(s ; z)=\mathcal{B}_{3}\left(s ; z_{a}-z\right)$. The states $\alpha=9$ ( $\alpha=11$ ) are then those with spin parallel (antiparallel) to $\mathcal{B}$. The renumbering is illustrated in Figure 5 , for the system in state $\alpha=9 ; 11$ within a field configuration path $C_{s}$ and in the corresponding state $\alpha=11 ; 9$ within $\bar{C}_{s}$.

\subsection{Dynamic and geometric phases}

The dynamical phases picked up by the states traversing the external field configurations are defined by the $z$-dependencies of their eigenenergies. In Figure 6 we show, for $s=1$, the real parts of the energies $E_{\alpha}(z)$ for $\alpha=9 ; 11,10,11 ; 9$, exhibiting the Zeeman- and Starkshifts according to the fields shown in Figure 2 As we can see from (73) of [11] the functional dependence of $E_{\alpha}(z)$ on the external fields is as follows:

$$
E_{\alpha}(z) \equiv E_{\alpha}\left(\mathcal{E}^{2}(z), \mathcal{B}^{2}(z),[\mathcal{E}(z) \cdot \mathcal{B}(z)]^{2}\right) .
$$

For our field configurations this can be simplified to

$$
E_{\alpha}(z)=E_{\alpha}\left(\left[\mathcal{E}_{1}(z)\right]^{2}, \mathcal{B}^{2}(z),\left[\mathcal{E}_{1}(z) \mathcal{B}_{1}(z)\right]^{2}\right) .
$$

We find, therefore, that in the ideal case where (34) holds the eigenenergies are the same, taking $s=1$, for the field path $C_{1}$ and the reverse path $\bar{C}_{1}$,

$$
\left.E_{\alpha}(z)\right|_{C_{1}}=\left.E_{\alpha}(z)\right|_{\bar{C}_{1}} .
$$

The same holds for the effective potential $\mathcal{V}_{\alpha}(z)$ because the additional geometric contributions are negligible, see (17) and (18),

$$
\left.\mathcal{V}_{\alpha}(z)\right|_{C_{1}}=\left.\mathcal{V}_{\alpha}(z)\right|_{\bar{C}_{1}}
$$

For the dynamic phases $\varphi_{\alpha}(z)$ we have, therefore, from (23) and (38) again in the ideal case

$$
\left.\varphi_{\alpha}(z)\right|_{C_{1}}=\left.\varphi_{\alpha}(z)\right|_{\bar{C}_{1}} .
$$

In (35)-39) we have

$$
\alpha \in\{9 ; 11,11 ; 9,10,12\} \text {. }
$$

In Figure 6 we show $\operatorname{Re} E_{\alpha}(z)$ for the realistic field configuration of Figure 2 where the symmetry relations (31) hold only approximately. In case that 31 would hold exactly the red curve $(\alpha=9 ; 11)$ would be the reflection of the blue curve $(\alpha=11 ; 9)$ on $z=z_{a} / 2$. We see that this reflection symmetry holds to a good approximation. The observed asymmetry in Figure 6 is caused mainly by the shift of $-\mathcal{B}_{3}^{\text {rev }}$ with respect to $\mathcal{B}_{3}$, see Figure 4, but does not qualitatively affect the main findings of this work. The asymmetry should rather be regarded as a realistic complication which our methods can easily deal with. The difference 


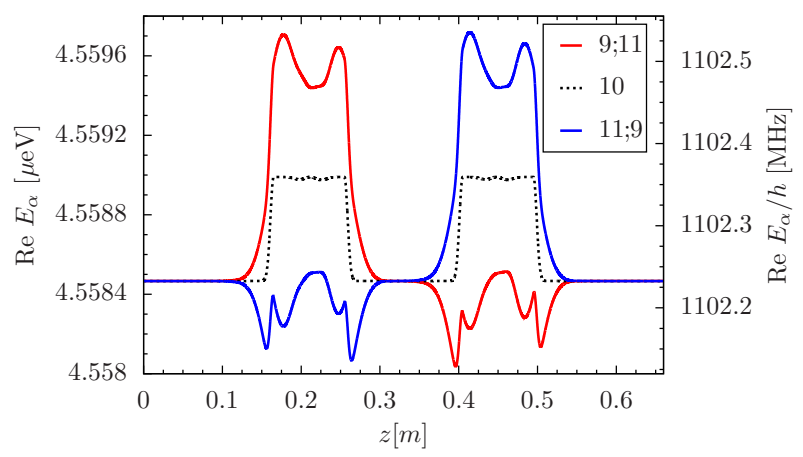

Figure 6: The real parts of the energies $E_{\alpha}(z)$ of the atomic states $\alpha=$ $9 ; 11,10$, and $11 ; 9$ in the fields shown in Figure 2 with $s=1$.

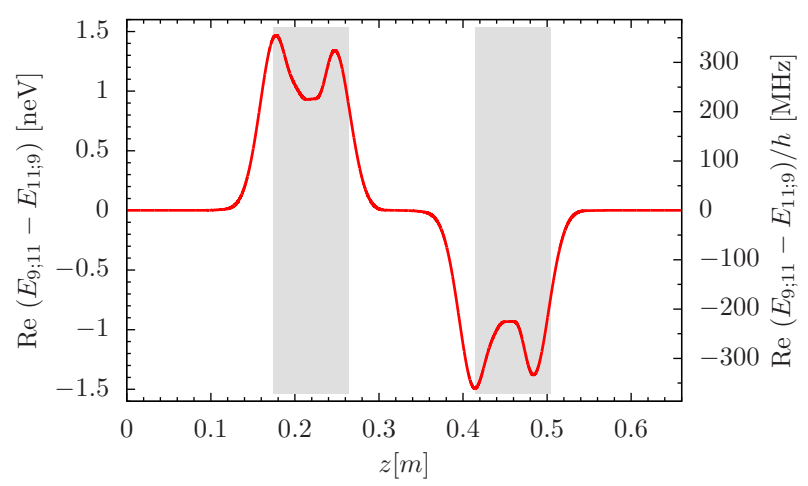

Figure 7: The real part of the energy difference 41 for the fields shown in Figure 2 with $s=1$. The shaded areas indicate the regions of nonzero electric fields.

$$
\operatorname{Re}\left[E_{9 ; 11}(z)-E_{11 ; 9}(z)\right]
$$

again for $s=1$, is shown in Figure 7. The adiabaticity conditions associated with these energy differences can be checked easily; see Appendix A.

In Figure 8 we show the $z$-dependent imaginary parts of the dynamic phase for the states $\alpha=9 ; 11$ and $11 ; 9$ exposed to the fields in Figure 2 where $s=1$. For these fields the imaginary parts of the dynamic phases are, within the accuracy of our numerical calculations, the same for $\alpha=9 ; 11$ and $\alpha=11 ; 9$. For the reverse field configuration (9), again with $s=1$ and in the ideal case where (34) holds, the imaginary parts of the dynamic phases are the same as for the original field configuration; see (39).

In Figure 9 we show the imaginary parts of the geometric phases, $\operatorname{Im} \gamma_{\alpha}(z)$, as functions of $z$ for $\alpha=9 ; 11$ and the curve $C_{1}$, and for $\alpha=11 ; 9$ and $\bar{C}_{1}$. A clear difference in $\operatorname{Im} \gamma_{\alpha}(z)$ between these two cases can be seen.

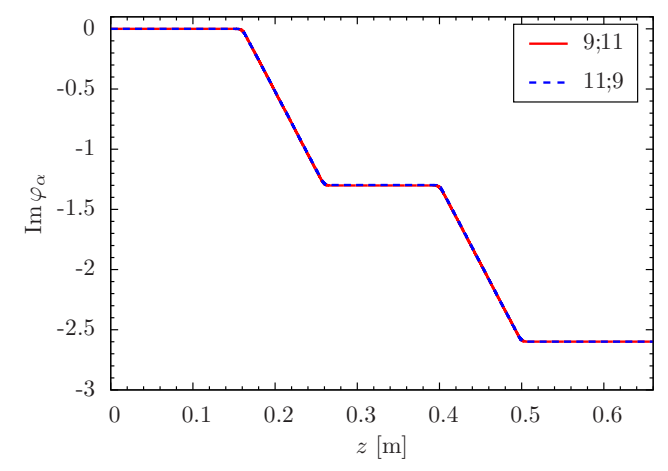

Figure 8: The imaginary parts of the dynamic phase 27, $\operatorname{Im} \varphi_{\alpha}(z)$, as function of $z$, for $s=1$, for the states $\alpha=9 ; 11$ and $11 ; 9$. The field configuration is given in Figure 2 The plot clearly shows where the imaginary parts of the dynamic phases are picked up along the $z$-axis.

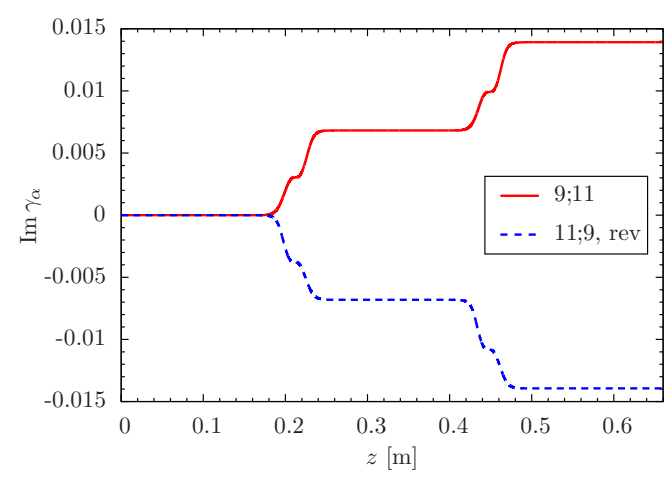

Figure 9: The imaginary part $\operatorname{Im} \gamma_{\alpha}(z)$ of the geometric phase, as function of $z$, for $s=1$, for the state $\alpha=9 ; 11$ in the field configuration path $C_{1}$ given in Figure 2 and for the state $\alpha=11 ; 9$ within the reversed configuration $\bar{C}_{1}$. The curves are identical for the states $\alpha=11 ; 9$, with $C_{1}$, and for $9 ; 11$, with $\bar{C}_{1}$.

For the curve $C_{1}$ the results for $\alpha=9 ; 11$ and 11;9 are the same. This is also the case for the curve $\bar{C}_{1}$. Note that here and in the following we compare $\alpha=9 ; 11(11 ; 9)$ in the field path $C_{s}$ to $\alpha=11 ; 9(9 ; 11)$ in the field path $\bar{C}_{s}$, thus taking into account the label change explained in Figure 5. The sign change of $\operatorname{Im} \gamma_{\alpha}\left(z_{a}\right)$ when going from $\alpha=9 ; 11$ and $C_{1}$ to $\alpha=11 ; 9$ and $\bar{C}_{1}$ in Figure 9 is clear from the property of geometric phases as line integrals. The fact that we have the same result for $\operatorname{Im} \gamma_{\alpha}\left(z_{a}\right)$ for $\alpha=9 ; 11$ and $11 ; 9$ is due to the special configuration of fields chosen; see Figures 2 and 3

We now turn to the difference of the imaginary parts of the dynamic and geometric phases. For the field configuration of Figure 2, corresponding to $s=1$ and the path $C_{1}$ in parameter space, this difference is shown in Figure 10 for $\alpha=9 ; 11$ and $\alpha=11 ; 9$. For the path 


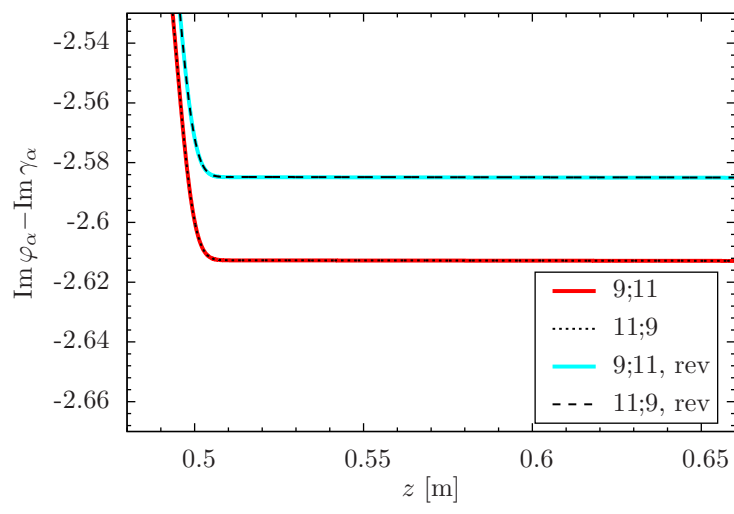

Figure 10: Combining the data shown in Figures 8 and 9 we depict $\operatorname{Im} \varphi_{\alpha}(z)-\operatorname{Im} \gamma_{\alpha}(z)$ for both the states $\alpha=9 ; 11$ and $\alpha=11 ; 9$ in $C_{1}$, together with the data obtained with the reversed path $\bar{C}_{1}$. The differences $\operatorname{Im} \varphi_{\alpha}\left(z_{a}\right)-\operatorname{Im} \gamma_{\alpha}\left(z_{a}\right)$ at the end of the interferometer are used in (42) to extract the lifetime modification (43, , 44.

$C_{1}$ and the reverse path $\bar{C}_{1}$ we see a clear difference in $\operatorname{Im} \varphi_{\alpha}(z)-\operatorname{Im} \gamma_{\alpha}(z)$. For the effective decay rates multiplied by the flight times, see [26], we get

$$
\begin{aligned}
T \Gamma_{9 ; 11, \mathrm{eff}}\left(C_{1}\right) & =T \Gamma_{11 ; 9, \mathrm{eff}}\left(C_{1}\right) \\
& =\left.\left(-2 \operatorname{Im} \varphi_{9 ; 11}\left(z_{a}\right)+2 \operatorname{Im} \gamma_{9 ; 11}\left(z_{a}\right)\right)\right|_{C_{1}} \\
& =2(2.599+0.0139), \\
T \Gamma_{9 ; 11, \mathrm{eff}}\left(\bar{C}_{1}\right) & =T \Gamma_{11 ; 9, \mathrm{eff}}\left(\bar{C}_{1}\right) \\
& =\left.\left(-2 \operatorname{Im} \varphi_{9 ; 11}\left(z_{a}\right)+2 \operatorname{Im} \gamma_{9 ; 11}\left(z_{a}\right)\right)\right|_{\bar{C}_{1}} \\
& =2(2.599-0.0139),
\end{aligned}
$$

if the symmetry condition $(31)$ is satisfied. The latter implies that a maximum revival, that is, a spin echo, can be observed at $s=1$, and the maxima of $\mathcal{F}_{p}\left(C_{s}\right)$ and $\mathcal{F}_{p}\left(\bar{C}_{s}\right)$ are both found at $s=1$. Furthermore, the same decay rates (42) are obtained for atomic states initially prepared in any superposition of $\alpha=9$ and $\alpha=11$. From the values (42) we obtain the ratio $R_{\alpha}$ of the fluxes of metastable hydrogen atoms in states $\alpha=11 ; 9$ and fieldpath $\bar{C}_{1}$ and $\alpha=9 ; 11$ and path $C_{1}$ as

$$
R_{9 ; 11}=\frac{\exp \left[-T \Gamma_{11 ; 9, \mathrm{eff}}\left(\bar{C}_{1}\right)\right]}{\exp \left[-T \Gamma_{9 ; 11, \mathrm{eff}}\left(C_{1}\right)\right]}=1.057 .
$$

Similarly we get

$$
R_{11 ; 9}=\frac{\exp \left[-T \Gamma_{9 ; 11, \mathrm{eff}}\left(\bar{C}_{1}\right)\right]}{\exp \left[-T \Gamma_{11 ; 9, \mathrm{eff}}\left(C_{1}\right)\right]}=1.057 .
$$

We expect the effect on the atomic lifetimes which is at the level of more than $5 \%$ to be accessible in a realistic

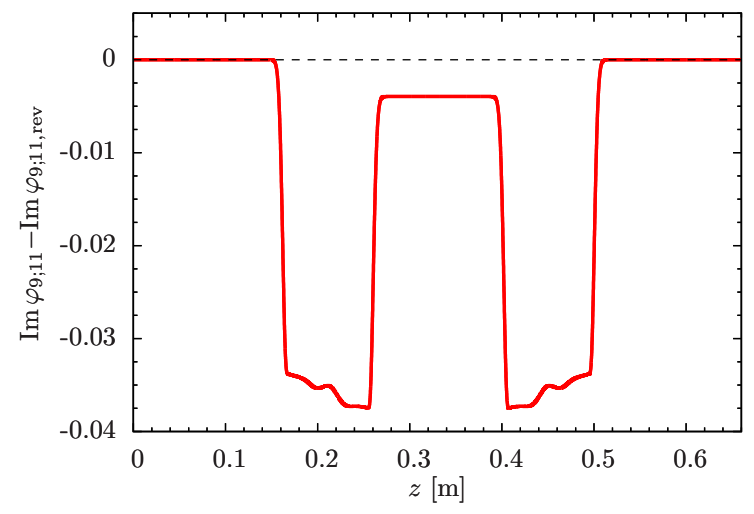

Figure 11: The difference $\operatorname{Im} \varphi_{9 ; 11}(z)-\operatorname{Im} \varphi_{9 ; 11, \text { rev }}(z)$ as a function of $z$, using the field configuration $C_{1}$ in Figure 2 and its reverse $\bar{C}_{1}$. This difference vanishes for fields obeying the symmetry condition (31) and is a measure for the violation of 39. As for spin echo signals, however, the $z$-dependence only enters at $z=z_{a}$, the violation of 39] is of no concern here. This makes our method rather robust with respect to imperfections in the experimental field configurations of the type 32.

experiment. However, $R_{\alpha}$ in (43), (44) is an appropriate measure of geometric lifetime modification only if a symmetric field configuration according to 31 is given. As we shall see below, the maxima of $\mathcal{F}_{p}\left(C_{s}\right)$ and $\mathcal{F}_{p}\left(\bar{C}_{s}\right)$ are in general found at different values of $s$ if (31) is not satisfied exactly. Although the norm of the atomic states $\alpha=9 ; 11$ and $\alpha=11 ; 9$ decays as obtained from (42), an initial superposition of $\alpha=9 ; 11$ and $\alpha=11 ; 9$ travelling through an asymmetric field configuration leads to interference patterns for which the maximal revival of the initial state is not reached at $s=1$. If the deviation from (31) were large enough, even completely destructive interference could be observed, misleadingly indicating large decay rates. Therefore, we cannot extract the lifetime modification for our slightly asymmetric realistic fields by only comparing $\mathcal{F}_{p}\left(C_{1}\right)$ and $\mathcal{F}_{p}\left(\bar{C}_{1}\right)$. Deviations from the symmetry conditions (31) occurring in realistic situations, however, do not affect the spin-echo measurements we are proposing here. To demonstrate this we show in Figure 11 the difference of the imaginary parts of $\varphi_{9 ; 11}(z)$ and $\varphi_{9 ; 11, \text { rev }}(z)$ for $s=1$ where the reversed fields are the realistic ones fulfilling (31) only approximately; see Figure 4. We see that $\operatorname{Im} \varphi_{9 ; 11}(z)-\operatorname{Im} \varphi_{9 ; 11 \text {,rev }}(z)$ is different from zero, but for $z=z_{a}$ the difference vanishes, since the integral over both regions I and II in $\sqrt{32}$ is the same. For our lifetime measurements only the value of these imaginary parts at $z=z_{a}$ matters and, therefore, our results (42), (43) and (44) hold unchanged also for our 


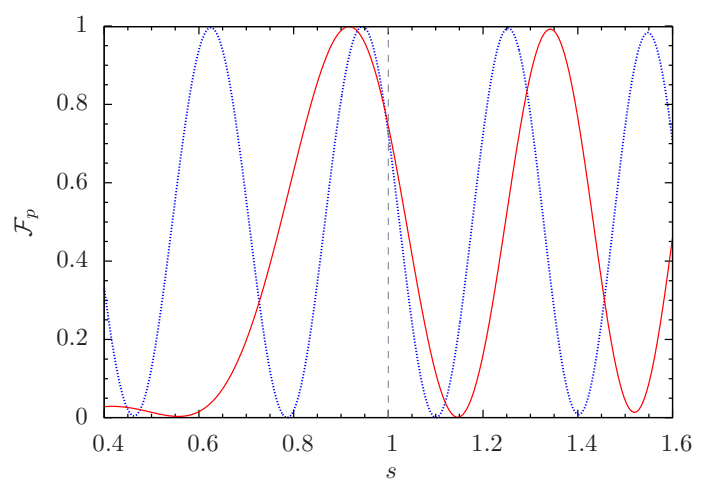

Figure 12: Spin echo integrated-flux curves for the paths $C_{s}$ (red solid line) and $\bar{C}_{s}$ (blue dotted line) using 30 and the field configuration in Figure 2 but with the electric field set to zero. Experimentally, $s$ can be varied by varying the current through the coil which generates the $\mathcal{B}_{3}$-field in the second (first) half of the interferometer for $C_{s}\left(\bar{C}_{s}\right)$. The vertical dashed line marks $s=1$. Without electric field the decay of the metastable states is negligible, and the spin echos reach almost unit amplitude for several values of $s$. However, the amplitudes also depend on the real parts of the $s$-dependent dynamic and geometric phases, as does the separation of the maxima along the $s$-axis.

realistic case where (31) is satisfied only approximately.

\subsection{Spin-echo measurement procedure}

We now turn to the actual measurement to be done with the spin-echo apparatus in order to extract the lifetime differences calculated above. A direct measurement of (43), (44) with the spin-echo field configuration in Figure 2 is possible by starting with hydrogen in the state $\alpha=9$ and projecting onto $\alpha=11$, i. e., $c_{9}=p_{11}=1$. The results obtained should then be compared to the case with reversed fields, starting with state $\alpha=11$ and projecting onto $\alpha=9$ at $z=z_{a}$. Notice, hereby, the change of labeling of the states at $z=z_{a} / 2$; see Figure 5 and the discussion after (34). However, aiming at an actual spinecho measurement, we propose to choose identical initial and analysing states, i. e., the superpositions in 30 .

Varying $s$, we obtain the spin-echo curves shown in Figures 12 and 13 These plots conveniently illustrate how lifetime modifications through geometric phases can be observed experimentally. The magnitude of this effect can be easily extracted by comparing the amplitudes of the spin-echo curves measured for $C_{s}$ and $\bar{C}_{s}$ as we discuss in more detail in the following.

Figure 14 shows the behaviour of $\mathcal{F}_{p}\left(C_{s}\right)$ and $\mathcal{F}_{p}\left(\bar{C}_{s}\right)$ near $s=1$ in an enlarged scale. The lifetime differences due to the differing imaginary parts of the geometric

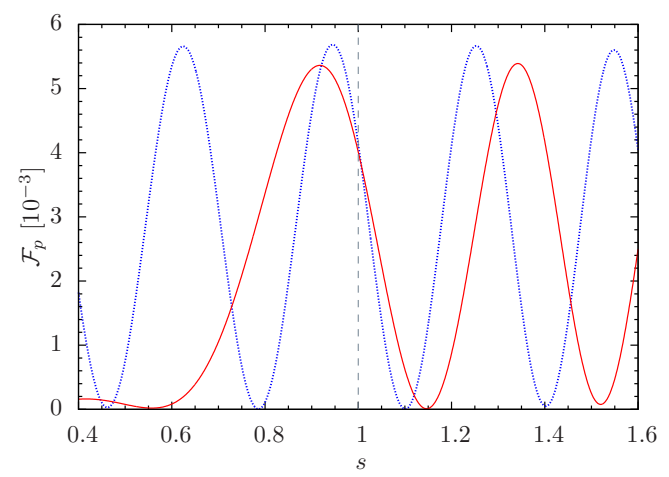

Figure 13: Spin echo curves for the paths $C_{s}$ (red solid line) and $\bar{C}_{s}$ (blue dotted line) using 30 as in Figure 12 but with the electric field turned on. The vertical dashed line marks $s=1$. The electric field results in decreased amplitudes of the spin echo curves, but the general shapes of the interference patterns are unchanged, cf. Figure 12 However, the presence of the electric field allows for the imaginary geometric phase to emerge after the closed path shown in Figure 3 has been traced out in parameter space, resulting in different values of the heights of maxima when comparing $C_{s}$ with $\bar{C}_{s}$. See Figure 14 for an enlarged display of the region around $s=1$.

phases for $C_{1}$ and $\bar{C}_{1}$ cause different spin echo curves for $C_{1}$ and $\bar{C}_{1}$. Note, however, that for a quantitative analysis we have to take into account also the real parts of the dynamic and geometric phases as will be explained below.

As our main result we predict that the amplitudes of the spin-echo signals obtained for $C_{s}$ and $\bar{C}_{s}$ differ due to imaginary geometric phases, to an extent that the effect is large enough to be experimentally accessible. The effect is extracted from the main features of the interference patterns $\mathcal{F}_{p}\left(C_{s}\right)$ and $\mathcal{F}_{p}\left(\bar{C}_{s}\right)$, with and without the electric field component $\mathcal{E}_{1}$ as shown in Figure 2, Comparing Figures 12 and 13 , we observe a decreased amplitude as the most pronounced effect of the electric field, while the phase of the interference patterns is not visibly affected, i. e., the electric field has negligible influence on the real parts of the geometric phases.

The frequencies of $\mathcal{F}_{p}\left(C_{s}\right)$ and $\mathcal{F}_{p}\left(\bar{C}_{s}\right)$ in Figure 12 with respect to $s$ are distinctly different, and both are $s$ dependent. As we will discuss in the following, the behavior of $\mathcal{F}_{p}$ as a function of $s$ is easily understood in terms of the $s$-dependent phases since the field configuration in Figure 2 allows for simplifications of the general expression (21). It will become clear that the different $s$-dependences of $\mathcal{F}_{p}\left(C_{s}\right)$ and $\mathcal{F}_{p}\left(\bar{C}_{s}\right)$ result from an interference effect involving the real parts of the geometric phases, while the different values of the maxima of $\mathcal{F}_{p}\left(C_{s}\right)$ and $\mathcal{F}_{p}\left(\bar{C}_{s}\right)$ originate mainly from the differences 


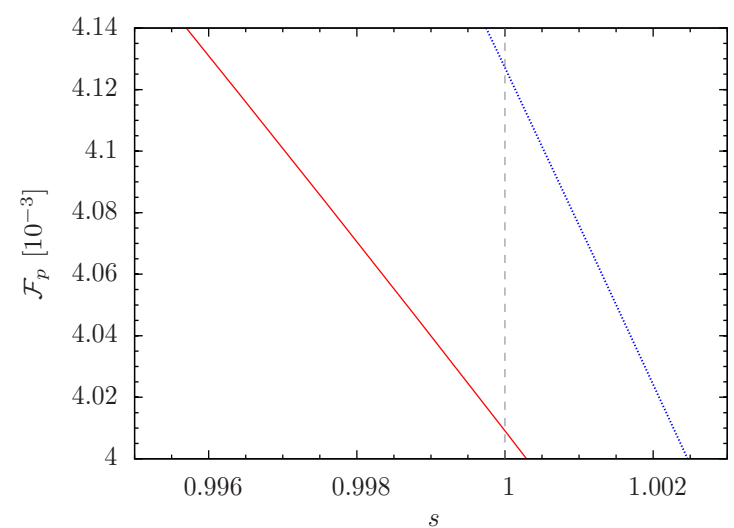

Figure 14: Magnification of the spin echo integrated-flux curves shown in Fig. 13 for the paths $C_{s}$ (red solid line) and $\bar{C}_{s}$ (blue dotted line) using (30) near $s=1$. The differing lifetimes specified in (42) show up as different values of the spin echo fluxes for $C_{1}$ and $\bar{C}_{1}$, respectively. However, while the reversed path gives a lower decay rate than the red path at $s=1$, the spin echo signal is not entirely determined by its amplitude, and we have to take into account the frequency of oscillation due to the cosine in 47 .

in the imaginary parts of the geometric phases.

As illustrated in Figure 15, the approximation

$$
\exp \left[-\left(\Delta \tau_{\beta}-\Delta \tau_{\alpha}\right)^{2} /\left(8 \sigma_{k}^{\prime 2}\right)\right] \approx 1
$$

holds at the percent level. Here $\Delta \tau_{\alpha}$ and $\sigma_{k}^{\prime}$ are the shifts of the reduced arrival times and the momentum-space widths of the wave packets defined in (99) and (86) of Ref. [9], respectively. Furthermore,

$$
\operatorname{Im}\left(\varphi_{9 ; 11}-\gamma_{9 ; 11}\right) \approx \operatorname{Im}\left(\varphi_{11 ; 9}-\gamma_{11 ; 9}\right)
$$

holds at the level of per mille. Hence, the flux 21) can be approximated by

$$
\begin{aligned}
& \mathcal{F}_{p}\left(C_{s}\right) \approx \frac{1}{2} \exp \left\{2 \operatorname{Im}\left[\varphi_{9 ; 11}\left(z_{a}\right)-\gamma_{9 ; 11}\left(z_{a}\right)\right]\right\} \\
& \times\left(1+\cos \left\{\operatorname{Re}\left[\varphi_{11 ; 9}\left(z_{a}\right)-\varphi_{9 ; 11}\left(z_{a}\right)\right]\right.\right. \\
&\left.\left.-\operatorname{Re}\left[\gamma_{11 ; 9}\left(z_{a}\right)-\gamma_{9 ; 11}\left(z_{a}\right)\right]\right\}\right)\left.\right|_{C_{s}}
\end{aligned}
$$

with a similar expression for $\mathcal{F}_{p}\left(\bar{C}_{s}\right)$,

$$
\begin{aligned}
\mathcal{F}_{p}\left(\bar{C}_{s}\right) \approx & \frac{1}{2} \exp \left\{2 \operatorname{Im}\left[\varphi_{11 ; 9}\left(z_{a}\right)-\gamma_{11 ; 9}\left(z_{a}\right)\right]\right\} \\
\times(1 & +\cos \left\{\operatorname{Re}\left[\varphi_{11 ; 9}\left(z_{a}\right)-\varphi_{9 ; 11}\left(z_{a}\right)\right]\right. \\
& \left.\left.-\operatorname{Re}\left[\gamma_{11 ; 9}\left(z_{a}\right)-\gamma_{9 ; 11}\left(z_{a}\right)\right]\right\}\right)\left.\right|_{\bar{C}_{s}}
\end{aligned}
$$

see Section 5.4 of [14]. In (48) we again make use of (46) but we now write $\operatorname{Im}(\varphi-\gamma)$ with index $11 ; 9$ to recall the

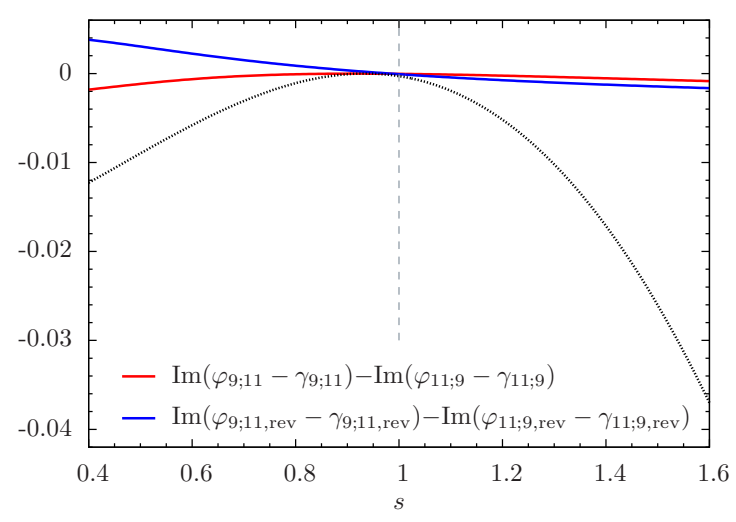

Figure 15: The relevant quantities that allow for the approximation 47) of 21, given as functions of $s$ for the field configuration in Figure 2 and its reverse. The black dotted line shows $\exp \left[-\left(\Delta \tau_{\beta}-\right.\right.$ $\left.\left.\Delta \tau_{\alpha}\right)^{2} /\left(8 \sigma_{k}^{\prime 2}\right)\right]-1$ for $C_{s}$ and $\beta=9 ; 11$ and $\alpha=11 ; 9$. The curves for $\beta=11 ; 9$ and $\alpha=9 ; 11$ as well as for the reversed path $\bar{C}_{s}$ are the same.

label change when going over from the curve $C_{s}$ to the reversed curve $\bar{C}_{s}$; see Figure 5 . The functions occuring in (47) and 48) have been calculated for the realistic field configurations of Figure 2 and are shown in Figures 16 and 17 for $C_{s}$ and $\bar{C}_{s}$, respectively. The results are close to fulfilling the symmetry relations

$$
\begin{aligned}
& \left.\operatorname{Re}\left[\varphi_{11 ; 9}\left(z_{a}\right)-\varphi_{9 ; 11}\left(z_{a}\right)\right]\right|_{C_{s}} \\
& \quad=\left.\operatorname{Re}\left[\varphi_{11 ; 9}\left(z_{a}\right)-\varphi_{9 ; 11}\left(z_{a}\right)\right]\right|_{\bar{C}_{s}}, \\
& \left.\operatorname{Re}\left[\gamma_{11 ; 9}\left(z_{a}\right)-\gamma_{9 ; 11}\left(z_{a}\right)\right]\right|_{C_{s}} \\
& \quad=-\left.\operatorname{Re}\left[\gamma_{11 ; 9}\left(z_{a}\right)-\gamma_{9 ; 11}\left(z_{a}\right)\right]\right|_{\bar{C}_{s}} .
\end{aligned}
$$

In the ideal case where (31) holds we would also expect

$$
\begin{aligned}
& \left.\operatorname{Re}\left[\varphi_{11 ; 9}\left(z_{a}\right)-\varphi_{9 ; 11}\left(z_{a}\right)\right]\right|_{C_{1}} \\
& \quad=\left.\operatorname{Re}\left[\varphi_{11 ; 9}\left(z_{a}\right)-\varphi_{9 ; 11}\left(z_{a}\right)\right]\right|_{\bar{C}_{1}}=0 \\
& \left.\operatorname{Re}\left[\gamma_{11 ; 9}\left(z_{a}\right)-\gamma_{9 ; 11}\left(z_{a}\right)\right]\right|_{C_{1}} \\
& \quad=\left.\operatorname{Re}\left[\gamma_{11 ; 9}\left(z_{a}\right)-\gamma_{9 ; 11}\left(z_{a}\right)\right]\right|_{\bar{C}_{1}}=0 .
\end{aligned}
$$

We see from Figures 16 and 17 that for realistic fields, the symmetry relations 49, 50 and 52) are rather well satisfied, but 51 not so well. We shall now expand the relevant functions around $s=1$ :

$$
\begin{gathered}
\left.\operatorname{Re}\left[\varphi_{11 ; 9}\left(z_{a}\right)-\varphi_{9 ; 11}\left(z_{a}\right)\right]\right|_{C_{s}} \\
=c_{\varphi}+m_{\varphi}(s-1)+\ldots, \\
\left.\operatorname{Re}\left[\varphi_{11 ; 9}\left(z_{a}\right)-\varphi_{9 ; 11}\left(z_{a}\right)\right]\right|_{\bar{C}_{s}}
\end{gathered}
$$




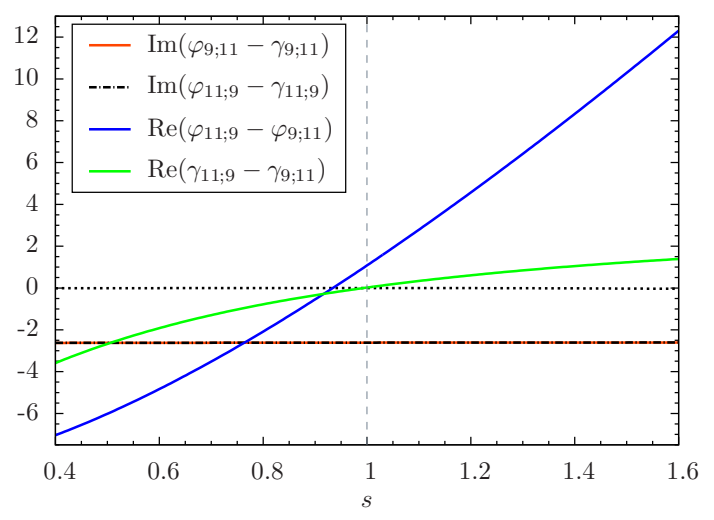

Figure 16: The real and imaginary parts of all combinations of phase differences that can occur between the atomic states $9 ; 11$ and $11 ; 9$, using the approximate expression 47 for $\mathcal{F}_{p}\left(C_{s}\right)$. The black dotted line shows $-\left(\Delta \tau_{\beta}-\Delta \tau_{\alpha}\right)^{2} /\left(8 \sigma_{k}^{\prime 2}\right)$ for $C_{s}$ and $\beta=9 ; 11$ and $\alpha=11 ; 9$. For our realistic field configurations $\varphi_{9 ; 11}\left(z_{a}\right)=\varphi_{11 ; 9}\left(z_{a}\right)$ holds only approximately. The $s$-dependent deviations of the dynamic phases from the spin echo point $\varphi_{9 ; 11}\left(z_{a}\right)=\varphi_{11 ; 9}\left(z_{a}\right)$ lead to the specific interference patterns in Figures 12 and 13

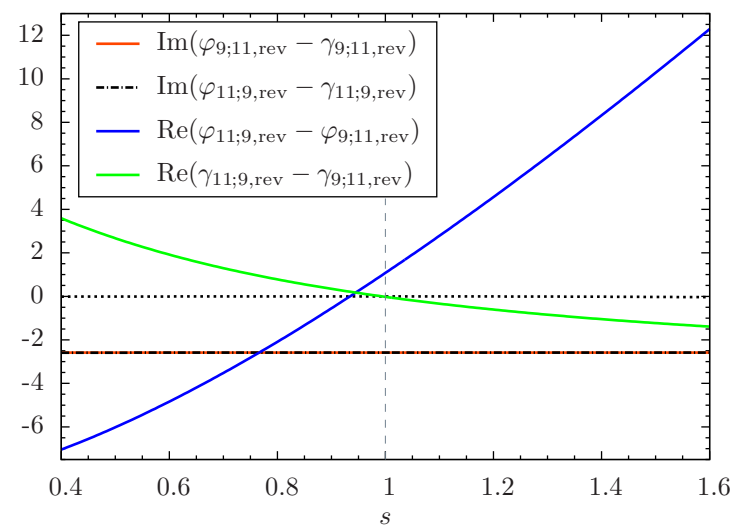

Figure 17: The relevant quantities that compose the approximate expression 47 of $\mathcal{F}_{p}\left(\bar{C}_{s}\right)$.

$$
\begin{gathered}
=\bar{c}_{\varphi}+\bar{m}_{\varphi}(s-1)+\ldots, \\
\left.\operatorname{Re}\left[\gamma_{11 ; 9}\left(z_{a}\right)-\gamma_{9 ; 11}\left(z_{a}\right)\right]\right|_{C_{s}} \\
=c_{\gamma}+m_{\gamma}(s-1)+\ldots, \\
\left.\operatorname{Re}\left[\gamma_{11 ; 9}\left(z_{a}\right)-\gamma_{9 ; 11}\left(z_{a}\right)\right]\right|_{\bar{C}_{s}} \\
=\bar{c}_{\gamma}+\bar{m}_{\gamma}(s-1)+\ldots .
\end{gathered}
$$

From Figures 16 and 17 we take that

$$
\begin{aligned}
& c_{\varphi} \approx \bar{c}_{\varphi}, \\
& m_{\varphi} \approx \bar{m}_{\varphi}, \\
& m_{\gamma} \approx-\bar{m}_{\gamma}, \\
& m_{\varphi}>m_{\gamma}>0,
\end{aligned}
$$

and

$$
c_{\gamma} \approx \bar{c}_{\gamma} \approx 0
$$

Keeping only the constant terms and those linear in $s-1$, which is a valid approximation when $|s-1| \lesssim 0.3$, we can approximate the cosine in (47), for $C_{s}$, as

$$
\cos \left[c_{\varphi}-c_{\gamma}+\left(m_{\varphi}-m_{\gamma}\right)(s-1)\right]
$$

and in 48 , for $\bar{C}_{s}$, as

$$
\cos \left[\bar{c}_{\varphi}-\bar{c}_{\gamma}+\left(\bar{m}_{\varphi}-\bar{m}_{\gamma}\right)(s-1)\right] .
$$

Let us first consider $s=1$ for which we get, from (47), (48), (57) and (58),

$$
\begin{aligned}
\mathcal{F}_{p}\left(C_{1}\right) \approx & \left.\frac{1}{2} \exp \left\{2 \operatorname{Im}\left[\varphi_{9 ; 11}\left(z_{a}\right)-\gamma_{9 ; 11}\left(z_{a}\right)\right]\right\}\right|_{C_{1}} \\
& \times\left[1+\cos \left(c_{\varphi}\right)\right] \\
\mathcal{F}_{p}\left(\bar{C}_{1}\right) \approx & \left.\frac{1}{2} \exp \left\{2 \operatorname{Im}\left[\varphi_{11 ; 9}\left(z_{a}\right)-\gamma_{11 ; 9}\left(z_{a}\right)\right]\right\}\right|_{\bar{C}_{1}} \\
& \times\left[1+\cos \left(\bar{c}_{\varphi}\right)\right]
\end{aligned}
$$

and for their ratio, using 42, 43, , 44), and $c_{\varphi} \approx \bar{c}_{\varphi}$,

$$
\begin{aligned}
\frac{\mathcal{F}_{p}\left(\bar{C}_{1}\right)}{\mathcal{F}_{p}\left(C_{1}\right)} \approx \frac{\exp \left[-T \Gamma_{9 ; 11, \mathrm{eff}}\left(\bar{C}_{1}\right)\right]}{\exp \left[-T \Gamma_{9 ; 11, \mathrm{eff}}\left(C_{1}\right)\right]} \\
=\frac{\exp \left[-T \Gamma_{11 ; 9, \mathrm{eff}}\left(\bar{C}_{1}\right)\right]}{\exp \left[-T \Gamma_{9 ; 11, \mathrm{eff}}\left(C_{1}\right)\right]}=1.057 .
\end{aligned}
$$

The discrepancy between this value for the quotient and

$$
\frac{\mathcal{F}_{p}\left(\bar{C}_{1}\right)}{\mathcal{F}_{p}\left(C_{1}\right)} \approx \frac{4.1271}{4.0091} \approx 1.0294,
$$

extracted from Figure 14 is due to the violation of 58 by our realistic field configuration,

$$
c_{\gamma} \approx-\bar{c}_{\gamma} \approx 0.022
$$

With $c_{\varphi} \approx \bar{c}_{\varphi} \approx 1.079$ (see Figures 16 and 17) we find, using (47), that

$$
\begin{aligned}
\frac{\mathcal{F}_{p}\left(\bar{C}_{1}\right)}{\mathcal{F}_{p}\left(C_{1}\right)} & \approx \frac{\exp \left[-T \Gamma_{11 ; 9, \mathrm{eff}}\left(\bar{C}_{1}\right)\right]}{\exp \left[-T \Gamma_{9 ; 11, \mathrm{eff}}\left(C_{1}\right)\right]} \frac{1+\cos \left(\bar{c}_{\varphi}-\bar{c}_{\gamma}\right)}{1+\cos \left(c_{\varphi}-c_{\gamma}\right)} \\
& \approx 1.0295
\end{aligned}
$$

consistent with (64). This observation underpins the necessity to measure the spin-echo curves for realistic field 
configurations over a sufficiently large $s$-range. In a followup experiment it will be necessary to make fits to $\mathcal{F}_{p}\left(C_{s}\right)$ and $\mathcal{F}_{p}\left(\bar{C}_{s}\right)$ and extract the imaginary parts of the geometric phases from these. We now show, that, e. g., the heights of the maxima of the spin-echo curves in Figure 13 can be used for this purpose. With (57)-60 we get, for $|s-1| \lesssim 0.3$, the approximate expressions

$$
\begin{aligned}
& \left.\mathcal{F}_{p}\left(C_{s}\right) \approx \frac{1}{2} \exp \left\{2 \operatorname{Im}\left[\varphi_{9 ; 11}\left(z_{a}\right)-\gamma_{9 ; 11}\left(z_{a}\right)\right]\right\}\right|_{C_{s}} \\
& \times\left\{1+\cos \left[c_{\varphi}-c_{\gamma}+\left(m_{\varphi}-m_{\gamma}\right)(s-1)\right]\right\}, \\
& \left.\mathcal{F}_{p}\left(\bar{C}_{s}\right) \approx \frac{1}{2} \exp \left\{2 \operatorname{Im}\left[\varphi_{11 ; 9}\left(z_{a}\right)-\gamma_{11 ; 9}\left(z_{a}\right)\right]\right\}\right|_{\bar{C}_{s}} \\
& \times\left\{1+\cos \left[c_{\varphi}-\bar{c}_{\gamma}+\left(m_{\varphi}+m_{\gamma}\right)(s-1)\right]\right\} .
\end{aligned}
$$

With $m_{\varphi}>m_{\gamma}>0$, see (57), we find that, near $s=1, \mathcal{F}_{p}\left(\bar{C}_{s}\right)$ should oscillate with higher frequency than $\mathcal{F}_{p}\left(C_{s}\right)$. We see from Figure 13 that this is indeed the case. At the maxima of $\mathcal{F}_{p}\left(C_{s}\right)$ and $\mathcal{F}_{p}\left(\bar{C}_{s}\right)$ that are the nearest to $s=1$ the cosines in (67) and (68) are equal to 1 and the ratio of the fluxes is determined by the effective lifetimes of the states. We find these maxima for $\mathcal{F}_{p}\left(C_{s}\right)$ $\left(\mathcal{F}_{p}\left(\bar{C}_{s}\right)\right)$ for $s=0.917(s=0.945)$ and $s=1.342$ $(s=1.253)$. For the ratios of the fluxes we get

$$
\begin{aligned}
& \frac{\exp \left[-T \Gamma_{11 ; 9, \mathrm{eff}}\left(\bar{C}_{1}\right)\right]}{\exp \left[-T \Gamma_{9 ; 11, \mathrm{eff}}\left(C_{1}\right)\right]} \approx \frac{\mathcal{F}_{p}\left(\bar{C}_{0.945}\right)}{\mathcal{F}_{p}\left(C_{0.917}\right)}=1.060, \\
& \frac{\exp \left[-T \Gamma_{11 ; 9, \mathrm{eff}}\left(\bar{C}_{1}\right)\right]}{\exp \left[-T \Gamma_{9 ; 11, \mathrm{eff}}\left(C_{1}\right)\right]} \approx \frac{\mathcal{F}_{p}\left(\bar{C}_{1.253}\right)}{\mathcal{F}_{p}\left(C_{1.342}\right)}=1.051 .
\end{aligned}
$$

As argued above, small uncertainties and asymmetries in a realistic experimental setup can lead to shifts and distortions of the spin-echo curves as compared to ideally symmetric field configurations. To extract the changes in atomic lifetimes at a desired confidence level, it is therefore in general not sufficient to measure the flux of atoms for a single value of $s$. We rather have to determine the spin-echo curves within a range of $s$ that includes the maxima of $\mathcal{F}_{p}\left(C_{s}\right)$ around $s=1$ and then invoke the same procedure for $\mathcal{F}_{p}\left(\bar{C}_{s}\right)$. Between $s=0.8$ and $s=1.4$ the two maxima of $\mathcal{F}_{p}\left(C_{s}\right)$ have approximately the same values, and the same holds for the maxima of $\mathcal{F}_{p}\left(\bar{C}_{s}\right)$. Therefore, both, the maxima for $s>1$ and $s<1$ serve to determine the geometry-induced relative changes in atomic lifetimes within the range $0.8 \lesssim s \lesssim 1.4$. We can regard the difference between (69) and $(70)$ as a rough measure of the uncertainty of our prediction for the geo-

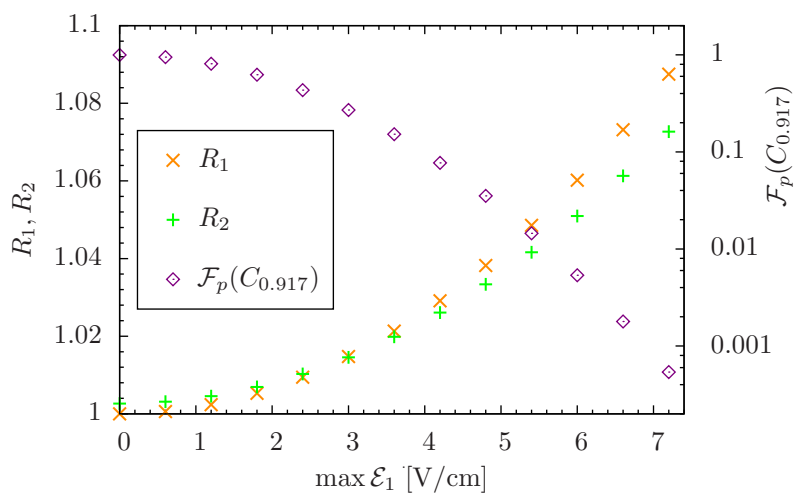

Figure 18: The ratios $R_{1}=\mathcal{F}_{p}\left(\bar{C}_{0.945}\right) / \mathcal{F}_{p}\left(C_{0.917}\right)$ and $R_{2}=$ $\mathcal{F}_{p}\left(\bar{C}_{1.253}\right) / \mathcal{F}_{p}\left(C_{1.342}\right)$ as well as the flux $\mathcal{F}_{p}\left(C_{0.917}\right)$ as a function of $\max \mathcal{E}_{1}$. The electric field in Figure 2 corresponds to $\max \mathcal{E}_{1}=$ $6 \mathrm{~V} / \mathrm{cm}$. At $\max \mathcal{E}_{1}=0$ we find $R_{1} \approx 1$. There, the corresponding maxima $\mathcal{F}_{p}\left(\bar{C}_{0.945}\right)$ and $\mathcal{F}_{p}\left(C_{0.917}\right)$ of the spin-echo curves, separated by $\Delta s \approx 0.028$ and relatively close to $s=1$, are practically of the same height. This is not the case for $R_{2}$ due to the fact that the maxima are found at $s$-values which differ significantly more, $\Delta s \approx 0.089$; cf. Figure 12 We see here that comparison of fluxes at different values of $s$ that are separated by large $\Delta s$ can increasingly mask the effect of the geometric phases on the decay rates. At which values of $s$ we can find the best estimate of the geometry-induced lifetime modification depends on the position of the maxima relative to $s=1$.

metric lifetime effects given the imperfections of a realistic field configuration. For other field configurations the quantities entering in $\mathcal{F}_{p}$ have to be investigated analogously to determine whether the lifetime modifications can be extracted from the maxima of the spin-echo curves.

We now study the dependence of the geometric lifetime effects on the applied electric field component $\mathcal{E}_{1}$. In the range where $\mathcal{E}_{1, \max } \leq 7.2 \mathrm{~V} / \mathrm{cm}$ we find that the maxima of the spin-echo curves, both for $\mathcal{F}_{p}\left(C_{s}\right)$ and $\mathcal{F}_{p}\left(\bar{C}_{s}\right)$, remain essentially at the same values of $s$ as extracted from Figure 13. We therefore show, in Figure 18, the ratios

$$
R_{1}=\frac{\mathcal{F}_{p}\left(\bar{C}_{0.945}\right)}{\mathcal{F}_{p}\left(C_{0.917}\right)}
$$

and

$$
R_{2}=\frac{\mathcal{F}_{p}\left(\bar{C}_{1.253}\right)}{\mathcal{F}_{p}\left(C_{1.342}\right)}
$$

at the same values of $s$ as in $(69)$ and $(70)$, respectively. We also show the absolute value of the spin-echo signal $\mathcal{F}_{p}\left(C_{0.917}\right)$ as a function of the magnitude of $\mathcal{E}_{1}$. The field $\mathcal{E}_{1}$ is scaled such that its maximum ranges between 0 and $7.2 \mathrm{~V} / \mathrm{cm}$. The ratio $R_{1}$ increases also for electric fields larger than $\mathcal{E}_{1, \max }=6 \mathrm{~V} / \mathrm{cm}$, but at the expense of 
the count rate which is proportional to $\mathcal{F}_{p}\left(C_{0.917}\right)$. We chose $\mathcal{E}_{1, \max }=6 \mathrm{~V} / \mathrm{cm}$, see Figure 2, as a reasonable compromise between the observable relative effect on the lifetimes and experimental feasibility.

The measurement of $\mathcal{F}_{p}$ can be considered as measurement of a random variable $\xi$ taking on two values. We set $\xi=1$ if an atom is detected at $z=z_{a}$ and $\xi=0$ if no atom is detected. In the latter case the atom may have decayed before arriving at $z_{a}$ or it may be in a state orthogonal to the analysing state $\mid p$ ) at $z_{a}$; cf. 20. Suppose now that we start with one atom at $z=0$. Then the probability to get $\xi=1$ is given by $\mathcal{F}_{p}$, the probability to get $\xi=0$ is $1-\mathcal{F}_{p}$. Thus, we have for the expectation value and the variance of $\xi$

$$
\begin{aligned}
E_{1}(\xi) & =\mathcal{F}_{p} \\
\operatorname{Var}_{1}(\xi) & =E_{1}\left(\xi^{2}\right)-\left[E_{1}(\xi)\right]^{2} \\
& =\mathcal{F}_{p}-\mathcal{F}_{p}^{2}=\mathcal{F}_{p}\left(1-\mathcal{F}_{p}\right) .
\end{aligned}
$$

Next we suppose that we start with $N$ atoms. Then we get for the average $\bar{\xi}$ the following expectation value and variance:

$$
\begin{aligned}
E_{N}(\bar{\xi}) & =\mathcal{F}_{p} \\
\operatorname{Var}_{N}(\bar{\xi}) & =\frac{1}{N} \operatorname{Var}_{1}(\xi)=\frac{1}{N} \mathcal{F}_{p}\left(1-\mathcal{F}_{p}\right) .
\end{aligned}
$$

If we want to measure $\mathcal{F}_{p}$ with a relative accuracy $\delta$ we should achieve

$$
\left[\operatorname{Var}_{N}(\bar{\xi})\right]^{1 / 2}<\delta E_{N}(\bar{\xi})
$$

that is,

$$
\frac{1}{N} \mathcal{F}_{p}\left(1-\mathcal{F}_{p}\right)<\delta^{2} \mathcal{F}_{p}^{2}
$$

This requires the number of atoms to obey

$$
N>\frac{1}{\delta^{2}}\left(\frac{1}{\mathcal{F}_{p}}-1\right)
$$

We consider, as a representative value of $\mathcal{F}_{p}$, half the maximum value $\mathcal{F}_{p}\left(C_{0.917}\right)$ at $\max \mathcal{E}_{1}=6 \mathrm{~V} / \mathrm{cm}$. From Figure 18 we then find $\mathcal{F}_{p}\left(C_{0.917}\right) / 2 \approx 2.7 \times 10^{-3}$. For a $0.5 \%$ measurement of this value of $\mathcal{F}_{p}$, condition 77 , requires us to work with

$$
N>1.5 \times 10^{7}
$$

atoms to obtain one data point on the spin-echo curve. To measure the complete spin-echo curves we will demand
100 data points for each, $C_{s}$ and $\bar{C}_{s}$. Hence, the total number of atoms needed is $N>3 \times 10^{9}$. With the corresponding accuracy of $0.5 \%$ per data point of $\mathcal{F}_{p}$ on the spin-echo curves ${ }^{2}$ it should be possible to obtain an accuracy of $10 \%$ for our geometric lifetime effect which is of the order of 5 to $6 \%$.

\section{Conclusions}

In this article we calculate the lifetime modification of metastable states of hydrogen due to geometric phases. A geometry-induced modification of atomic decay rates has not been observed experimentally thus far. In addition to imaginary dynamic phases, which emerge in an effective description of decaying atomic states travelling in an adiabatic way through electromagnetic fields, the hydrogen state vectors acquire imaginary geometric phases in suitable chiral electromagnetic field configurations. We use the time evolution of a superposition of metastable states propagating in a field configuration which is based on realistic experimental conditions to compute the flux of atoms arriving at the detector of a longitudinal atomicbeam spin-echo apparatus. We analyse the relevant quantities entering the description of the propagating atomic wave packet, in particular the dynamic and geometric phases, and propose a realistic scheme to observe the change of lifetimes experimentally. We ensure adiabatic evolution in spatial regions where geometric phases for the hydrogen state vectors emerge. We vary the field configuration to obtain spin-echo curves which are conveniently accessible in experiment. We show in detail how to extract the geometry-induced change of lifetime from the maxima of the spin-echo curves and estimate the necessary number of metastable atoms to be $4 \times 10^{9}$ for a statistically significant measurement. We find that the lifetime is modified at the level of $5 \%$ due to geometric phases. We estimate that this effect is large enough to be observed under realistic experimental conditions.

${ }^{2}$ The theoretical error of $\mathcal{F}_{p}$ is estimated to be of the same order; see [9]. 


\section{Appendix}

\section{A Conditions for adiabatic evolution of the states}

Employing the field configuration from Figure 2 with $s=1$, we find that the adiabaticity conditions (B.16) and (B.22) from [8] for the field variations are satisfied. We get

$$
\begin{gathered}
\frac{1}{\mathcal{E}_{0}} \max _{t \in[0, T]}\left|\frac{\partial \mathcal{E}}{\partial t}\right|<\frac{1}{T} \longleftrightarrow 0.69 \lesssim 1, \\
\frac{1}{\mathcal{B}_{0}} \max _{t \in[0, T]}\left|\frac{\partial \mathcal{B}}{\partial t}\right|<\frac{1}{T} \longleftrightarrow 0.1 \lesssim 1,
\end{gathered}
$$

with $\mathcal{E}_{0}=477.3 \mathrm{~V} / \mathrm{cm}, \mathcal{B}_{0}=43.65 \mathrm{mT}, T=\left(z_{a}-\right.$ $\left.z_{0}\right) / v_{z}$. Wherever geometric phases emerge along the $z$-axis the energy separation $\Delta E$ between the involved states is large enough for adiabatic evolution:

$$
\Delta z \gtrsim \frac{h v_{z}}{\Delta E} \longleftrightarrow 90 \mathrm{~mm} \gg 19 \mathrm{~mm}
$$

see (27) from [9]. For $\Delta z$ we have $90 \mathrm{~mm}$ from the fields of Figure 2 Of course, for $s>1$ the adiabaticity condition A.3 is satisfied as well, whereas $s$ may not be taken much smaller than $s=1$.

\section{B Field configuration}

We employ a field configuration as depicted in Figure 2 which is actually available in the laboratory. The magnetic field components are fits to measured data. The electric field component $\mathcal{E}_{1}(z)$ is calculated via a finiteelements method and is experimentally realisable with an appropriate set of capacitor plates. It is straightforward to adjust the analysis presented in this work for slightly different experimental realisations of $\mathcal{E}_{1}(z)$. The remaining field components are chosen to be zero, the electric field is given in units of $\mathrm{V} / \mathrm{cm}$, the magnetic field components in units of $\mu \mathrm{T}$. For the calculation of $\mathcal{F}_{p}\left(C_{s}\right)$ and $\mathcal{F}_{p}\left(\bar{C}_{s}\right)$ with $C_{1}$ illustrated in Figure 2, we vary $s$ in the $z$-intervals $[0.32330919,0.66]$ and $[0,0.33669081]$, respectively. We define

$$
\begin{aligned}
z_{m} & =0.33380917, \\
z_{1} & =0.31676777, \\
z_{2} & =0.57813638 .
\end{aligned}
$$

Using $c_{0}=-15625, c_{1}=0.009, c_{2}=0.0105, c_{3}=$ $0.07, c_{4}=0.08, c_{5}=0.16, c_{6}=0.17$, and employing the syntax 'A ? B : C' for 'B to be true if A is, and C to be true if A is not' and use logical 'AND' and 'OR', the fields are given as

\footnotetext{
-
}

$$
\begin{aligned}
\mathcal{E}_{1}(z)= & \left\{\left(z+c_{1}>z_{m}-c_{5} \text { AND } z+c_{1}<z_{m}-c_{3}\right) \text { OR }\left(z+c_{1}>z_{m}+c_{4} \text { AND } z+c_{1}<z_{m}+0.17\right) ? 1:\right. \\
& \exp \left[c_{0}\left(z+c_{1}-\left(z_{m}-c_{5}\right)\right)\left(z+c_{1}-\left(z_{m}-c_{5}\right)\right)\right]+\exp \left[c_{0}\left(z+c_{1}-\left(z_{m}-c_{3}\right)\right)\left(z+c_{1}-\left(z_{m}-c_{3}\right)\right)\right] \\
& +\exp \left[c_{0}\left(z+c_{1}-\left(z_{m}+c_{4}\right)\right)\left(z+c_{1}-\left(z_{m}+c_{4}\right)\right)\right]+\exp \left[c_{0}\left(z+c_{1}-\left(z_{m}+c_{6}\right)\right)\left(z+c_{1}-\left(z_{m}+c_{6}\right)\right)\right] \\
& +\left(z_{a}+c_{1}-z>z_{m}-c_{5} \text { AND } z_{a}+c_{1}-z<z_{m}-c_{3}\right) \text { OR }\left(z_{a}+c_{1}-z>z_{m}+c_{4} \text { AND } z_{a}+c_{1}-z<z_{m}+c_{6}\right) ? 1: \\
& \exp \left[c_{0}\left(z_{a}+c_{1}-z-\left(z_{m}-c_{5}\right)\right)\left(z_{a}+c_{1}-z-\left(z_{m}-c_{5}\right)\right)\right]+\exp \left[c_{0}\left(z_{a}+c_{1}-z-\left(z_{m}-c_{3}\right)\right)\left(z_{a}+c_{1}-z-\left(z_{m}-c_{3}\right)\right)\right] \\
& \left.+\exp \left[c_{0}\left(z_{a}+c_{1}-z-\left(z_{m}+c_{4}\right)\right)\left(z_{a}+c_{1}-z-\left(z_{m}+c_{4}\right)\right)\right]+\exp \left[c_{0}\left(z_{a}+c_{1}-z-\left(z_{m}+c_{6}\right)\right)\left(z_{a}+c_{1}-z-\left(z_{m}+c_{6}\right)\right)\right]\right\} \\
\mathcal{B}_{1}(z)= & z+c_{2}<z_{m} ?\left\{z+c_{2}<z_{1} ?-153.283 \exp \left[-2251.75\left(z+c_{2}-0.221739\right)^{2}\right] \sin \left[3.95282\left(z+c_{2}-0.222097\right)\right]\right. \\
& -50.294 \exp \left[-2174.46\left(z+c_{2}-0.457696\right)^{2}\right] \sin \left[11.8633\left(z+c_{2}-0.193543\right)\right]: \\
& {\left[z+c_{2}<0.36223008 ? 0:\left\{z+c_{2}<z_{2} ?-153.283 \exp \left[-2251.75\left(z+c_{2}-0.221739\right)^{2}\right] \sin \left[3.95282\left(z+c_{2}-0.222097\right)\right]\right.\right.} \\
& \left.\left.\left.-50.294 \exp \left[-2174.46\left(z+c_{2}-0.457696\right)^{2}\right] \sin \left[11.8633\left(z+c_{2}-0.193543\right)\right]: 0\right\}\right]\right\}: \\
& \left\{z+c_{2}<z_{1} ? 153.283 \exp \left[-2251.75\left(z+c_{2}-0.221739\right)^{2}\right] \sin \left[3.95282\left(z+c_{2}-0.222097\right)\right]\right. \\
& 50.294 \exp \left[-2174.46\left(z+c_{2}-0.457696\right)^{2}\right] \sin \left[11.8633\left(z+c_{2}-0.193543\right)\right]: \\
& {\left[z+c_{2}<0.36223008 ? 0:\left\{z+c_{2}<z_{2} ? 153.283 \exp \left[-2251.75\left(z+c_{2}-0.221739\right)^{2}\right] \sin \left[3.95282\left(z+c_{2}-0.222097\right)\right]\right.\right.} \\
& \left.\left.\left.50.294 \exp \left[-2174.46\left(z+c_{2}-0.457696\right)^{2}\right] \sin \left[11.8633\left(z+c_{2}-0.193543\right)\right]: 0\right\}\right]\right\}
\end{aligned}
$$




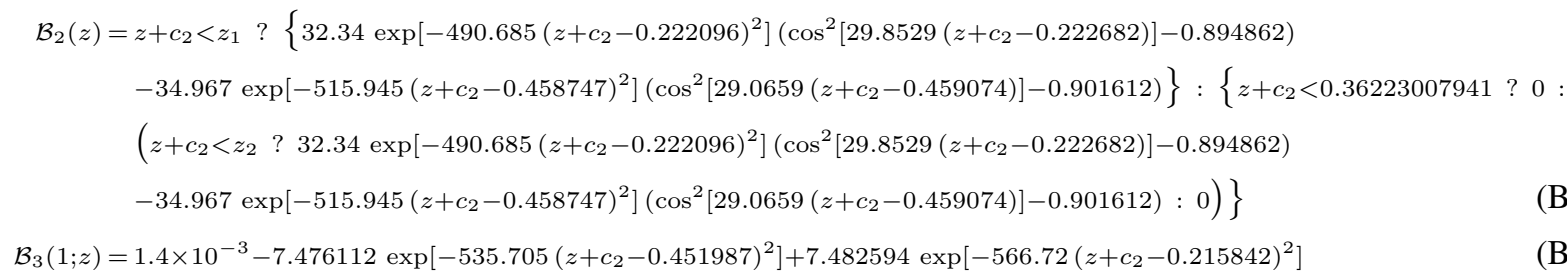

\section{References}

[1] M. V. Berry, Quantal phase factors accompanying adiabatic changes, Proc. R. Soc. Lond. A 392, 45 (1984).

[2] B. Simon, Holonomy, the quantum adiabatic theorem, and Berry's phase, Phys. Rev. Lett. 51, 2167 (1983).

[3] J. C. Garrison and E. M. Wright, Complex geometrical phases for dissipative systems, Phys. Lett. A 128, 177 (1988).

[4] Ch. Miniatura, C. Sire, J. Baudon and J. Bellissard, Geometrical Phase Factor for a Non-Hermitian Hamiltonian, Europhys. Lett. 13, 199 (1990), Correction: Europhys. Lett. 14, 91 (1991).

[5] S. Massar, Applications of the complex geometric phase for metastable systems, Phys. Rev. A 54, 4770 (1996).

[6] M. V. Berry, Physics of Nonhermitian Degeneracies, Czech. J. Phys. 54, 1039 (2004).

[7] T. Bergmann, T. Gasenzer and O. Nachtmann, Metastable states, the adiabatic theorem and parity violating geometric phases I, Eur. Phys. J. D 45, 197 (2007).

[8] T. Bergmann, T. Gasenzer and O. Nachtmann, Metastable states, the adiabatic theorem and parity viol- ating geometric phases II, Eur. Phys. J. D 45, 211 (2007).

[9] T. Bergmann, M. DeKieviet, T. Gasenzer, O. Nachtmann and M.-I. Trappe, Parity Violation in Hydrogen and Longitudinal Atomic Beam Spin Echo I, Eur. Phys. J. D 54, 551 (2009).

[10] M. DeKieviet, T. Gasenzer, O. Nachtmann and M.-I. Trappe, Longitudinal atomic beam spin echo experiments: a possible way to study parity violation in hydrogen, Hyperfine Interact. 200, 35 (2011).

[11] T. Gasenzer, O. Nachtmann and M.-I. Trappe, Metastable states of hydrogen: their geometric phases and flux densities, Eur. Phys. J. D 66, 113 (2012).

[12] M. DeKieviet, D. Dubbers, C. Schmidt, D. Scholz and U. Spinola, ${ }^{3} \mathrm{He}$ spin echo: A new atomic beam technique for probing phenomena in the neV range, Phys. Rev. Lett. 75, 1919 (1995).

[13] T. Bergmann, Theorie des longitudinalen Atomstrahl-Spinechos und paritätsverletzende BerryPhasen in Atomen, $\mathrm{PhD}$ thesis, Ruprecht-Karls-Universität, Heidelberg (2006).

[14] M.-I. Trappe, Parity-Violating and Parity-Conserving Berry Phases for Hydrogen and Helium in an Atom Interferometer, PhD thesis, Ruprecht-KarlsUniversität, Heidelberg (2012). 